\title{
APLICAÇÃO DA METODOLOGIA DE SUPERFÍCIE DE RESPOSTA NA OTIMIZAÇÃO DA MISTURA DE ANTIOXIDANTES COM EFEITO SOBRE A ESTABILIDADE LIPÍDICA DO OVO ATOMIZADO
}

\author{
MICHELLE GARCÊZ DE CARVALHO ${ }^{*}$ \\ ALFREDO TENUTA FILHO ${ }^{2}$
}

\begin{abstract}
O ovo por ser um alimento rico em colesterol e lipídios, tornase susceptível à oxidação, principalmente quando submetido a processos que envolvem altas temperaturas, como é o caso da atomização. A prevenção ou redução da oxidação lipídica do ovo é possível pelo uso de antioxidantes. Os objetivos deste trabalho foram: a) Verificar por meio de teste de concentração de antioxidantes, a estabilidade lipídica do ovo integral pasteurizado atomizado adicionado da mistura de extrato de alecrim, extrato de chá verde e $\mathrm{BHA}(\mathrm{AO})$; e b) Otimizar a concentração da mistura de $\mathrm{AO}$ adicionada ao ovo líquido integral pasteurizado, posteriormente atomizado, pelo modelo matemático proposto pela Metodologia de Superfície de Resposta (RSM). Foram ensaiadas dez misturas de antioxidantes no ovo integral pasteurizado atomizado (OIPA) e refletiu na sua estabilidade oxidativa lipídica, verificada pelas TBARS (substâncias reativas ao ácido tiobarbitúrico), CATL (capacidade antioxidante total da fração lipídica), CT-F (capacidade redutora pelo reativo de Folin - Ciocalteau), AGL (ácidos graxos livres) e AS-233 (substâncias absorvidas a $233 \mathrm{~nm}$ ). Os resultados mostraram que a RSM foi adequada para descrever a formação dos AGL e AS-233 no OIPA, podendo estas últimas serem usadas para fins preditivos. A otimização da mistura de antioxidantes baseou-se no modelo matemático obtido com as AS-233, onde foi proposto como sistema antioxidante $150 \mathrm{ppm}$ de BHA, 600ppm de alecrim e $300 \mathrm{ppm}$ de chá verde. A RSM foi determinante para se constatar com precisão aceitável a influência das diferentes concentrações dos antioxidantes adicionados ao ovo para manter sua estabilidade lipídica.
\end{abstract}

PALAVRAS-CHAVE: OVO ATOMIZADO, OXIDAÇÃO, ANTIOXIDANTES, METODOLOGIA DE SUPERFÍCIE DE RESPOSTA

1 Doutora, Professora do curso de nutrição da Universidade Federal de Sergipe. *correspondência: M.G de Carvalho (michellegarcezpi@hotmail.com)

2 Doutor, Professor do curso de farmácia da Universidade de São Paulo, eetenuta@usp.br 


\section{INTRODUÇÃO}

O ovo é consumido diretamente como alimento, na sua forma in natura, e/ou indiretamente, como ingrediente participando da formulação de outros produtos alimentares, depois de processados (TAI; CHEN; CHEN, 2000; OHATA; VIOTTO, 2011). Os principais processamentos industriais utilizados em ovo são a pasteurização e a atomização (KITAHARA, 2004).

A pasteurização tem sido utilizada como processamento prévio à atomização, garantindo a estabilidade necessária do ovo líquido como matéria-prima a ser transformada em ovo em pó (FELLOWS, 2006). A atomização consiste da pulverização do produto líquido em um compartimento que recebe fluxo de ar quente. Como consequência há evaporação da água e a separação do produto em pó. A rápida evaporação da água permite manter baixa a temperatura das partículas sólidas, de maneira que a alta temperatura do ar de secagem $\left(170-230^{\circ} \mathrm{C}\right)$ não afete demasiadamente $\mathrm{O}$ produto (ROSA; TSUKADA; FREITAS, 2010).

O ovo por ser um alimento rico em colesterol e lipídios, torna-se susceptível à oxidação, principalmente quando submetido a processos que envolvem altas temperaturas, como é o caso da atomização. Assim há a aceleração das reações entre os lipídios e oxigênio molecular, resultando na formação de compostos indesejáveis, como dialdeído malônico, óxidos de colesterol e produtos da reação de Maillard (GALOBART et al., 2001; CABONI et al., 2005; MAZALLI; BRAGAGNOLO, 2007).

O controle da estabilidade lipídica é necessário para prevenir a perda nutricional, o comprometimento de atributos sensoriais e a produção de compostos tóxicos. A prevenção ou redução da oxidação lipídica no ovo atomizado e demais alimentos, pode ser possível pelo uso de antioxidantes (GALOBART et al., 2001; LAROSA, 2011; BERTOLIN et al., 2010; 2011).

Estudos indicam que há maior interesse da indústria de alimentos pelos antioxidantes naturais, devido à tendência dos consumidores em rejeitar antioxidantes sintéticos em alimentos e à comprovação dos efeitos colaterais (alergia e doenças degenerativas) (BERTOLIN et al., 2010) ao organismo dos antioxidantes sintéticos em doses elevadas (ZHENG; WANG, 2001; RIBEIRO; SERAVALLI, 2004; RAMALHO; JORGE, 2006; FACCO; LAGE; GODOY, 2009; BERTOLIN et al., 2011). Assim, diversos extratos de ervas como alecrim, chá verde, coentro, sálvia, tomilho e manjericão têm sido investigados devido o seu poder antioxidante, que é atribuído ao seu conteúdo de compostos fenólicos (ANGELO; JORGE, 2007; PERUMALLA; NAVAM, 2011). Extratos de alecrim e chá verde têm sido utilizados na substituição parcial de antioxidantes sintéticos convencionais, associados ou não entre si (CARVALHO, 2012).

Sendo inevitável o uso não totalmente seguro de antioxidantes sintéticos, como vem ocorrendo com o BHA (butilato de hidroxianisol) e BHT (butilato de hidroxitolueno), para impedir as consequências provocadas pela oxidação lipídica em alimentos, torna-se importante substituí-los, ainda que parcialmente, por antioxidantes naturais, que sejam seguros, e cuja ação possa no mínimo ser semelhante. Dessa forma, o presente estudo buscou por meio da metodologia de superfície de resposta (RSM) avaliar os efeitos de múltiplos fatores e suas interações, em uma ou mais variáveis de resposta, cujos objetivos foram: a) verificar por meio de teste de concentração de antioxidantes, a estabilidade lipídica do ovo integral pasteurizado atomizado adicionado da mistura de extrato de alecrim, extrato de chá verde e BHA; e b) otimizar a concentração da mistura de extrato de alecrim, extrato de chá verde e BHA a ser adicionada ao ovo líquido integral pasteurizado, posteriormente atomizado, pelo modelo matemático proposto pela Metodologia de Superfície de Resposta.

\section{MATERIAL E MÉTODOS}

\subsection{REAGENTES E AMOSTRAS}


Os reagentes utilizados tinham grau analítico compatível, adquiridos de vários fornecedores: hexano (Synth, 147), sulfato de sódio anidro (Vetec, S2032.01.AG), ácido sulfúrico (Synth, 31190), molibdato de amônio (Vetec, 122), fosfato de sódio monobásico (Vetec, 0752), etanol (Vetec, 000103.06), a-tocoferol (Sigma, T3251), n-Hexano, grau HPLC (Sigma-Aldrich, 110-54-3), Isopropanol, grau HPLC(Sigma-Aldrich, 67-63-0), Padrão de 7-cetocolesterol (3ß-hidroxicolest-5a-ene7-one) - Sigma (C2394), Florisil - Merck (MgO:SiO2, 15:85), acetona (Vetec, 187), metanol (Vetec, 102), ácido tiobarbitúrico (Merck, 1.08180.0025), reativo de Folin Ciocalteu (Dinâmica, 2336), ácido tricloroacético (Vetec, 466), clorofórmio (Vetec, 192), n-heptano (Synth, H.1001.01.BJ), ácido gálico (Sigma-Aldrich, G7384), fenolftaleína (Synth, F1005. 01. AE), hidróxido de sódio (Synth, H2000. 01. $A G)$, antioxidantes naturais da Danisco: Extrato de chá Verde - Guardian ${ }^{\mathrm{TM}}{ }^{\mathrm{M}}$ Green Tea Extract $20 \mathrm{M}$ e Extrato de alecrim - Guardian ${ }^{\mathrm{TM}}$ Rosemary Extract 08; e BHA (butilato de hidroxianisol) - Synth (B1028.02.AE).

As amostras de ovo in natura (em casca) recém produzido (3 dias) foram adquiridas em supermercados na cidade de São Paulo-SP. Foram ensaiadas amostras em triplicata $(\mathrm{n}=$ 3) compreendendo 12 ovos/amostra, isentos de casca, as quais ficaram estocadas na própria embalagem comercial, mantidas em temperatura ambiente $\left(25^{\circ} \mathrm{C} \pm 2\right)$ até o início dos experimentos (até 2 dias). 0 prazo de estocagem correspondeu ao de validade de 30 dias indicado pelo fabricante.

\subsection{OBTENÇÃO DO OVO LÍQUIDO INTEGRAL, OVO LÍQUIDO INTEGRAL PASTEURIZADO E OVO INTEGRAL PASTEURIZADO ATOMIZADO}

Ovos (in natura) sem casca foram homogeneizados com auxílio de bastão de vidro, em um béquer revestido externamente com papel-alumínio, e peneirados em tela de náilon $\left(1 \mathrm{~mm}^{2}\right)$. A amostra obtida foi transferida para um processador de alimentos (Philips Walita 600 WATT), mantido sob vácuo, e agitada por 1 minuto para completa homogeneização entre a clara e a gema. A amostra homogeneizada foi transferida para um kitassato, aplicado vácuo por meio de uma bomba de vácuo (Quimis Q355B), para que assim houvesse a remoção de bolhas de ar formadas anteriormente, e mantendo o sistema sob banho de gelo para acelerar esse processo (ESCARABAJAL, 2011).

$\mathrm{O}$ ovo líquido integral, obtido como anteriormente descrito, foi pasteurizado em banhomaria a efetivamente a $65^{\circ} \mathrm{C}$, por 3 minutos, seguido da redução imediata da temperatura (choque térmico), por imersão em banho de gelo até $0^{\circ} \mathrm{C}$ (ESCARABAJAL, 2011).

O ovo líquido integral pasteurizado resfriado obtido anteriormente foi adicionado de três partes de água destilada, misturado com auxílio de um bastão de vidro e então submetido à atomização em escala de laboratório (Buchi Mini Spray Dryer B-290), nas seguintes condições empregadas: temperatura de entrada de $170^{\circ} \mathrm{C}$; temperatura de saída de $63^{\circ} \mathrm{C}$; vazão de alimentação, de $15 \mathrm{~mL} /$ min.; fluxo do atomizador, de 439L/h; e, tempo de operação de 1h/L (ESCARABAJAL, 2011).

\subsection{DESENHO EXPERIMENTAL}

Por meio de teste de concentração de antioxidantes, usando a metodologia de superfície de resposta (RSM), foram adicionados antioxidantes (Extrato de alecrim, Extrato de chá verde e BHA) em diferentes concentrações ao ovo líquido integral pasteurizado, antes da atomização. Adotou-se um delineamento experimental completo do tipo $2^{3}$, com 2 replicatas no ponto central, totalizando 11 ensaios independentes (MONTGOMERY, 2000), como é mostrado na Tabela 1. Na Tabela 2 encontram-se os níveis das variáveis independentes a serem testadas em misturas. Todos os ensaios foram realizados em triplicata.

Não foi encontrada legislação específica para adição de extratos de alecrim e chá verde em alimentos. O fornecedor (Danisco) recomenda que sejam adicionados de $0,1 \mathrm{~g}$ a $0,5 \mathrm{~g}$ chá verde/ $\mathrm{Kg}$ de óleo ou gordura e de $0,2 \mathrm{~g}$ a $1 \mathrm{~g}$ de alecrim/ $\mathrm{Kg}$ de óleo ou gordura (DANISCO, 2011). Portanto, as concentrações dos extratos de alecrim e chá verde encontram-se dentro dos limites recomendados 
pela DANISCO (2011), enquanto que a concentração do BHA está de acordo com a legislação brasileira (BRASIL, 2005).

A concentração em ppm dos antioxidantes adicionados ao ovo líquido integral pasteurizado baseou-se na concentração de lipídios totais do ovo líquido integral. O produto atomizado obtido foi mantido a $5 \pm 2^{\circ} \mathrm{C}$ por até dois dias, até à realização das análises.

\section{TABELA 1. DELINEAMENTO EXPERIMENTAL ADOTADO}

\begin{tabular}{cccccccccccc}
\hline Antioxidantes & \multicolumn{10}{c}{ Ensaios } \\
\cline { 2 - 12 } & $\mathbf{1}$ & $\mathbf{2}$ & $\mathbf{3}$ & $\mathbf{4}$ & $\mathbf{5}$ & $\mathbf{6}$ & $\mathbf{7}$ & $\mathbf{8}$ & $\mathbf{9}$ & $\mathbf{1 0}$ & $\mathbf{1 1}^{*}$ \\
\hline Alecrim & -1 & -1 & -1 & -1 & 1 & 1 & 1 & 1 & 0 & 0 & - \\
Chá verde & -1 & -1 & 1 & 1 & -1 & -1 & 1 & 1 & 0 & 0 & - \\
BHA & -1 & 1 & -1 & 1 & -1 & 1 & -1 & 1 & 0 & 0 & - \\
\hline Sem antioxidante & & & & & & & & & & &
\end{tabular}

TABELA 2. NÍVEIS DAS VARIÁVEIS INDEPENDENTES A SEREM TESTADAS

\begin{tabular}{|c|c|c|c|c|c|c|c|c|}
\hline \multicolumn{3}{|c|}{ Alecrim (ppm) } & \multicolumn{3}{c|}{ Chá verde (ppm) } & \multicolumn{3}{c|}{ BHA (ppm) } \\
\hline-1 & 0 & 1 & -1 & 0 & 1 & -1 & 0 & 1 \\
\hline 200 & 600 & 1000 & 100 & 300 & 500 & 100 & 150 & 200 \\
\hline
\end{tabular}

\subsection{OTIMIZAÇÃO DAS CONCENTRAÇÕES DA MISTURA DE ANTIOXIDANTES}

A otimização da concentração da mistura de antioxidantes procedeu-se com o uso da função de desejabilidade, que seguiu a imposição computacional exaustiva nos pontos com respostas ótimas (DERRINGER; SUICH, 1980). Assim, novas amostras de ovo líquido integral pasteurizado foram preparadas e a elas adiciona a uma nova combinação de antioxidantes definida pelo programa estatístico como ótima, em seguida foram atomizadas e submetidas aos mesmos protocolos experimentais para averiguar a adequabilidade dos modelos em seu poder preditivo e $o$ valor previsto.

\subsection{QUANTIFICAÇÕES ANALÍTICAS}

\subsubsection{Lipídios Totais}

Feito por gravimetria, após a extração com clorofórmio/metanol $(2: 1, \mathrm{v} / \mathrm{v})$, segundo o método de Folch, Lees e Stanley (1957), adaptado por Csallany et al. (1989), com adição de $\mathrm{NaCl} 0,88 \%$, no caso do ovo líquido, com posterior eliminação do solvente $\left(32^{\circ} \mathrm{C}\right.$, sob vácuo) em rotaevaporador (TECNAL) e aquecimento em estufa a $105^{\circ} \mathrm{C} / 40$ minutos.

\subsubsection{Avaliação da capacidade antioxidante total da fração lipídica pelo método do fosfomolibdênio}

O procedimento analítico foi realizado com base em Mohamed, Pineda, Aguilar (2007); Pietro, Pineda, Aguilar (1999) e Carvalho (2012). Os resultados foram expressos em equivalentes em $\mathrm{mg}$ de $\alpha$-tocoferol/g. 


\subsubsection{Quantificação de Substâncias Reativas ao Ácido Tiobarbitúrico - TBARS}

O método empregado para quantificação das substâncias reativas ao ácido tiobarbitúrico foi o de (VYNCKE, 1970), que propõe a extração da amostra com ácido tricloroacético, seguido pela reação com o ácido tiobarbitúrico e leitura do complexo formado em espectrofotômetro, a $538 \mathrm{~nm}$. Os resultados foram expressos em mg dialdeído malônico/kg.

\subsubsection{Capacidade redutora pelo reativo de Folin-Ciocalteau (CR-F) - Fenólicos totais}

A extração dos fenólicos totais foi feita segundo Medina (2009) e a quantificação pelo método de Zhang et al. (2006). Os resultados foram expressos em $\mathrm{mg}$ de ácido gálico/g.

A metodologia contemplou a extração da fração lipídica (Folch; Lees; Stanley, 1957; Csallany et al., 1989), seguida da determinação titulométrica da porcentagem dos ácidos graxos livres, expressos em porcentagem de ácido oléico/g de lipídio (ANVISA, 2005).

\subsubsection{Quantificação das substâncias absorvidas a 233nm (AS-233)}

Para a quantificação das substâncias absorvidas a 233nm, a extração lipídica seguiu o que foi indicado por Folch, Lees, Stanley (1957). A extração das AS-233 foi segundo Penazzi et al. (1995) e a detecção realizada em espectrofotômetro de UV-Visível 1800 (SHIMADZU) a 233nm. Os resultados foram expressos em equivalente em $\mu \mathrm{g}$ 7-cetocolesterol/ mg lipídio.

\subsection{ANÁLISE ESTATÍSTICA}

Empregou-se a metodologia de superfície de resposta (RSM), através do programa estatístico SAS Institute (1997) para avaliar o efeito combinado das variáveis independentes sobre as variáveis dependentes, calcular os coeficientes de regressão $\left(R^{2}\right)$, fazer a análise de variância (ANOVA) e construir as superfícies de resposta, com nível de significância de $5 \%$.

A otimização da concentração da mistura de antioxidantes procedeu-se com o uso da função de desejabilidade, que seguiu a imposição computacional exaustiva nos pontos com respostas ótimas (DERRINGER; SUICH, 1980). O modelo matemático obtido no valor experimental foi submetido a duas metodologias estatísticas: i) teste t-Student pareado e ii) cálculo do erro absoluto residual usando a equação: Erro absoluto residual (\%) $=[($ Valor experimental - Valor previsto)/Valor experimental] x 100, para então ser otimizada a concentração dos antioxidantes adotados.

\section{RESULTADOS E DISCUSSÃO}

\subsection{EFEITOS DA ADIÇÃO E CONCENTRAÇÃO DA MISTURA DE ANTIOXIDANTES SOBRE A ESTABILIDADE LIPÍDICA DO OVO}

A RSM é uma forma eficaz do método estatístico que é útil para modelação matemática (WU et al., 2012), obtendo-se modelos preditivos em função das variáveis independentes e as variáveis de resposta (MOURA et al., 2007). Pode também ser efetivamente usada como uma ferramenta para encontrar a combinação das variáveis independentes que produza uma resposta ótima (SAHA et al., 2011).

Foram avaliados os efeitos da adição e concentração dos antioxidantes extrato comercial de alecrim, extrato comercial de chá verde e BHA (realizada antes da atomização), sobre o ovo integral pasteurizado atomizado (OIPA), por meio da metodologia de superfície de resposta (RSM). 
A estabilidade lipídica do OIPA foi avaliada pelas variáveis de resposta - capacidade antioxidante total da fração lipídica (CATL), substâncias reativas ao ácido tiobarbitúrico (TBARS), capacidade redutora pelo reativo Folin - Ciocalteau (CR-F), ácidos graxos livres (AGL) e substâncias absorvidas a 233nm (AS-233). Foram ensaiadas dez misturas dos antioxidantes mencionados, com monitoramento de um controle (sem adição de antioxidante) (Tabela 3). Os resultados obtidos variaram consideravelmente, refletindo a influência das diferentes concentrações dos três antioxidantes usados sobre a estabilidade oxidativa lipídica do ovo integral pasteurizado atomizado (Tabela 3).

Tabela 3. Efeitos da adição e concentração das misturas de antioxidantes (Alecrim/ Chá verde/BHA/) ao ovo integral pasteurizado atomizado avaliados pela capacidade antioxidante total da fração lipídica (CATL), substâncias reativas ao ácido tiobarbitúrico (TBARS), capacidade redutora pelo reativo de Folin - Ciocalteau (CR-F), ácidos graxos livres (AGL) e substân$\begin{array}{ccccc}\text { cias } & \text { absorvidas } & \text { a } & 233 \mathrm{~nm}\end{array}$

\begin{tabular}{|c|c|c|c|c|c|c|c|c|}
\hline \multirow[b]{2}{*}{ 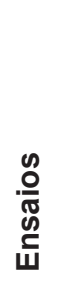 } & \multicolumn{3}{|c|}{$\begin{array}{c}\text { Variáveis } \\
\text { independentes }\end{array}$} & \multicolumn{5}{|c|}{ Variáveis de resposta ${ }^{1}$} \\
\hline & $\begin{array}{l}\frac{E}{\frac{E}{U}} \\
\frac{d}{<}\end{array}$ & 胥 $\frac{0}{0}$ & 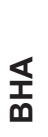 & CATL & TBARS & CR-F & AGL & AS-233 \\
\hline 1 & -1 & -1 & -1 & $3,306^{a}$ & $0,00127^{c}$ & $0,0030^{\mathrm{cd}}$ & $3,863^{\mathrm{bc}}$ & $2,883^{a b}$ \\
\hline 2 & -1 & -1 & 1 & $3,430^{\mathrm{a}}$ & $0,00088^{\text {de }}$ & $0,0028^{\text {def }}$ & $3,147^{c}$ & $2,407^{\mathrm{abc}}$ \\
\hline 3 & -1 & 1 & -1 & $2,420^{\mathrm{bc}}$ & $0,00126^{\mathrm{cd}}$ & $0,0039^{b}$ & $5,663^{a}$ & $2,863^{a b}$ \\
\hline 4 & -1 & 1 & 1 & $1,879^{\text {cde }}$ & $0,00167^{b}$ & $0,0029^{\text {cde }}$ & $3,043^{c}$ & $1,210^{c}$ \\
\hline 5 & 1 & -1 & -1 & $1,706^{\mathrm{de}}$ & $0,00197^{\mathrm{ab}}$ & $0,0025^{\text {deg }}$ & $5,003^{\mathrm{ab}}$ & $1,570^{\mathrm{bc}}$ \\
\hline 6 & 1 & -1 & 1 & $1,959^{\text {cde }}$ & $0,00080^{\text {ef }}$ & $0,0021^{\mathrm{g}}$ & $3,897^{\mathrm{bc}}$ & $1,587^{\mathrm{bc}}$ \\
\hline 7 & 1 & 1 & -1 & $2,860^{\mathrm{ab}}$ & $0,00210^{a}$ & $0,0052^{a}$ & $3,413^{c}$ & $1,743^{\mathrm{bc}}$ \\
\hline 8 & 1 & 1 & 1 & $2,114^{\text {cde }}$ & $0,00097^{\text {ce }}$ & $0,0025^{\text {deg }}$ & $5,740^{\mathrm{a}}$ & $1,395^{c}$ \\
\hline 9 & 0 & 0 & 0 & $1,542^{\mathrm{e}}$ & $0,00047^{f}$ & $0,0034^{\mathrm{bc}}$ & $3,760^{\mathrm{bc}}$ & $1,930^{\mathrm{bc}}$ \\
\hline 10 & 0 & 0 & 0 & $1,680^{\mathrm{de}}$ & $0,00047^{f}$ & $0,0023^{f g}$ & $3,820^{\mathrm{bc}}$ & $2,560^{\mathrm{abc}}$ \\
\hline \multicolumn{4}{|c|}{$\begin{array}{l}\text { Controle negativo } \\
\text { antioxidantes) }\end{array}$} & $2,245^{c d}$ & $0,00110^{\text {ce }}$ & $0,0024^{\mathrm{efg}}$ & $3,483^{c}$ & $2,963^{a}$ \\
\hline \multicolumn{4}{|c|}{ Desvio padrão conjunto } & 0,653 & 0,00054 & 0,0009 & 0,991 & 0,00054 \\
\hline \multicolumn{4}{|c|}{$p$-valor ${ }^{*}$} & 0,0695 & 0,3422 & 0,8266 & 0,4186 & 0,3422 \\
\hline \multicolumn{4}{|c|}{ p-valor ${ }^{\#}$} & $<0,001$ & $<0,001$ & $<0,001$ & $<0,001$ & $<0,001$ \\
\hline
\end{tabular}

Concentraçōes dos antioxidantes: $200 \mathrm{ppm}(-1)-600 \mathrm{ppm}(0)-1000 \mathrm{ppm}(1)$ alecrim; 100ppm (-1) - 300ppm (0) - 500ppm (1) chá verde; 100ppm (-1) - 150ppm (0) - 200ppm (1) BHA. ${ }^{1}$ Resultados médios $(n=3)$ expressos em base seca. Letras diferentes na mesma coluna representam resultados estatisticamente diferentes $(p<0,05)$ de acordo com o teste de Tukey ou Kruscal-Wallis. CATL: equivalente em mg de $\alpha$-tocoferol/g; TBARS: $\mathrm{mg}$ de dialdeído malônico/kg; CR-F: $\mathrm{mg}$ de ácido gálico/g; AGL: porcentagem de ácido oléico/g lipídio; AS-233: equivalente em $\mu$ g 7-cetocolesterol/ mg lipídio. ${ }^{*}$ Valor-p para teste de homogeneidade de variâncias de Hartley (F-max); \#Valor-p para a análise de variância univariada ou teste de Kruscal-Wallis.

Nos ensaios 1, 2 e 7 a disponibilidade da CATL aumentou 147,26; 152,78 e 127,39\%, respectivamente, em relação ao controle, enquanto no ensaio 9 houve redução de $37,04 \%$, possibilitando que as TBARS ficassem reduzidas em $57,27 \%$. O aumento da CATL sugere a participação da mistura dos antioxidantes adicionados e, a redução das TBARS, um sinal de proteção antioxidante. 
Nos ensaios 5 e 7 ficaram evidentes aumentos de 179,10 e 190,91\% das TBARS, respectivamente, em relação ao controle, caracterizando a oxidação de ácidos graxos. Essas ocorrências, contudo, não vieram acompanhadas do comprometimento perceptível da CATL correspondente disponível. A ineficiência dos antioxidantes envolvidos nos ensaios 5 e 7 poderiam justificar o aumento das TBARS.

Semelhante ao verificado na Tabela 3, Guardiola et al. (1997) investigou o efeito da adição da mistura de antioxidantes como o palmitato de ascorbila e o $\boldsymbol{\alpha}$-tocoferol, nas concentrações de 100 e 200ppm, em ovo atomizado, e observou que os antioxidantes usados não conseguiram evitar a perda de ácidos graxos poli-insaturados e a oxidação do colesterol. Medina (2009) verificou que a adição de 300ppm de extrato comercial de tocoferóis não impediu o aumento das TBARS em ovo atomizado comercial.

A capacidade redutora pelo reativo de Folin - Ciocalteau (CR-F) (Tabela 3) foi maior no ensaio 7, podendo ter havido correlação com uma maior concentração de antioxidantes naturais, alecrim (1000ppm) e chá verde (500ppm), que são fontes reconhecidas de compostos fenólicos responsáveis por tal característica (MORAIS et al., 2009; AFONSO; SANTANA; MANCINI-FILHO, 2010) e uma menor concentração do BHA (100ppm). Os compostos fenólicos têm sido muito estudados devido à sua influência na qualidade dos alimentos pela inibição da peroxidação lipídica (DEGÁSPARI; WASZCZYNSKYJ, 2004).

A quantidade dos ácidos graxos livres (AGL) verificados nos ensaios 3, 5 e 8 (Tabela 3) aumentaram em 162,50; 143,64 e 164,80\% respectivamente, em relação ao controle. Esse fato sugere que a combinação dos antioxidantes nas concentrações empregadas não foram efetivas para minimizar a hidrólise dos lipídios produzindo AGL. A decomposição acelerada dos lipídios quando submetidos ao aquecimento (NAWAR, 2000), como ocorreu na atomização, poderia justificar o aumento de AGL. Adicionalmente, o processo oxidativo lipídico é quase sempre acompanhado pela formação de AGL. Com a atomização (industrial) do ovo líquido integral pasteurizado, Medina (2009) constatou uma redução de $A G L$ de $73,5 \%$. A redução observada foi associada à volatilização de ácidos graxos e/ou oxidação com posterior conversão em outros produtos (TOMPKINS; PERKINS, 2000).

De modo geral, a oxidação dos ácidos graxos ocorre mais rapidamente com a elevação da temperatura, devido à redução parcial da solubilidade do oxigênio entre a água e os lipídios. Os ácidos graxos oxidam-se mais rapidamente ainda na forma livre do que na conjugada na molécula de acilglicerol (NAWAR, 2000; MEDINA, 2009).

Os ensaios 8 e 4 exibiram forte redução das substâncias absorvidas a 233nm (AS-233), entre 52,92 a $59,16 \%$, em relação ao controle, pressuposta pela ação dos antioxidantes envolvidos. As substâncias com absorção máxima a $233 \mathrm{~nm}$ (com é o caso do 7-cetocolesterol), ou parcial (não identificadas no presente trabalho) (Chen, Chen, 1994), foram quantificadas como AS-233 e expressas em equivalentes de 7-cetocolesterol. Como já apontado, o 7-cetocolesterol é considerado o óxido mais frequentemente encontrado como produto da oxidação do colesterol, sendo proposto que a sua ocorrência sirva para caracterizar tal evento (Tabela 3).

A adição ao ovo dos antioxidantes (BHA, BHT, TBHQ, extrato de alecrim, tocoferóis, galato de propila, palmitato de ascorbila) antes ou após a atomização, não foi eficiente para reduzir e/ou erradicar completamente a oxidação do colesterol, podendo está associado à volatilização desses durante a atomização (MORGAN; ARMSTRONGAN, 1987; HUBER; PIKE; HUBER, 1995; LAl et al., 1995; MEDINA, 2009; ESCARABAJAL, 2011).

A oxidação lipídica não pode ser estimada apenas por simples ensaios in vitro. No entanto, alguns compostos mostraram uma alta correlação entre os seus efeitos antioxidantes, quando aplicados diretamente no alimentos e quando avaliados por metodologias in vitro (CAPITANI et al., 2009). Além disso, o comportamento dos antioxidantes em mistura pode ser diferente daquele quando o antioxidante é testado individualmente (ANGELO; JORGE, 2008; CAPITANI et al., 2009). A influência das variáveis independentes (Alecrim/Chá verde/ BHA) sobre as variáveis de 
respostas (CATL, TBARS, CR-F, AGL e AS-233), relativas ao do ovo integral pasteurizado atomizado, como consta na Tabela 3, é observada na Tabela 4, apoiada no delineamento experimental completo do tipo $2^{3}$, adotado pelo coeficiente de regressão significativo $(p<0,05)$, equação de regressão, valor de $p$ da regressão, coeficiente de determinação $\left(R^{2}\right)$ e valor de $p$ (falta de ajuste) do modelo matemático.

TABELA 4. ANÁLISES DE REGRESSÃO DOS RESULTADOS CONTIDOS NA TABELA 3, REFERENTES À CAPACIDADE ANTIOXIDANTE TOTAL DA FRAÇÃO LIPÍDICA (CATL), SUBSTÂNCIAS REATIVAS AO ÁCIDO TIOBARBITÚRICO (TBARS), CAPACIDADE REDUTORA PELO REATIVO DE FOLIN - CIOCALTEAU (CR-F), ÁCIDOS GRAXOS LIVRES (AGL) E SUBSTÂNCIAS ABSORVIDAS A 233NM (AS-233)

\begin{tabular}{|c|c|c|c|c|}
\hline $\begin{array}{l}\text { Variável de } \\
\text { resposta }\end{array}$ & Equação de regressão & $\begin{array}{c}\mathrm{p} \text {-valor } \\
\text { (regressão) }\end{array}$ & $\mathbf{R}^{2}(\%)$ & $\begin{array}{c}\text { p-valor } \\
\text { (falta de } \\
\text { ajuste) }\end{array}$ \\
\hline CATL & $y=2,2896+0,4683 X_{2}{ }^{*} X_{3}$ & 0,0085 & 41,23 & 0,1249 \\
\hline TBARS & $\begin{array}{l}\text { Nenhum coeficiente do modelo de regressão foi } \\
\text { significativo }(p<0,05)\end{array}$ & 0,01 & NA & 0,2978 \\
\hline CR-F & $\begin{array}{l}\text { Nenhum coeficiente do modelo de regressão foi } \\
\text { significativo }(p<0,05)\end{array}$ & 0,0494 & NA & 0,7068 \\
\hline AGL & $\begin{array}{l}Y=4,135-0,2646 X_{1}+0,2921 X_{2}+0,2437 X_{3}+ \\
0,1912 X_{1}{ }^{*} X_{3}+0,5696 X_{1}{ }^{*} X_{2}+0,6671 X_{1}{ }^{*} X_{2}{ }^{*} X_{3}\end{array}$ & 0,002 & 93,59 & 0,0567 \\
\hline AS-233 & $y=2,014-0,3077 X_{1}-0,3835 X_{2}$ & 0,0444 & 63,74 & 0,6930 \\
\hline
\end{tabular}

$\mathrm{X}_{1}=\mathrm{BHA} ; \mathrm{X}_{2}=$ Alecrim; $\mathrm{X}_{3}=$ Chá verde. $\mathrm{R}^{2}=$ Coeficiente de determinação. $\mathrm{NA}=$ Não aplicável. CATL: equivalente em $\mathrm{mg}$ de $\alpha$-tocoferol/ g; TBARS: $\mathrm{mg}$ de dialdeído malônico/kg; CR-F: mg de ácido gálico/g; AGL: porcentagem de ácido oléico/g lipídio; AS-233: equivalente em $\mu \mathrm{g}$ 7-cetocolesterol/ mg lipídio.

Pela equação de regressão (Tabela 4) pode-se observar que apenas o chá verde e alecrim influenciaram a CATL, ou seja, a interação desses antioxidantes aumenta significativamente a CATL, mas apesar de apresentar coeficiente do modelo de regressão e valor de $p$ (regressão) significativos $(p<0,05)$ e falta de ajuste não significativo $(p>0,05)$, o modelo matemático tem baixo poder de explicação $\left(R^{2}<60 \%\right)$, por isso não pode ser usado para fins preditivos.

Segundo a Tabela 4, para as TBARS e CR-F os modelos matemáticos não mostraram ser apropriados para descrever o efeito da mistura dos antioxidantes em ovo integral pasteurizado 
atomizado, usando as condições experimentais adotadas no presente estudo, uma vez que os coeficientes do modelo de regressão não foram significativos $(p<0,05)$, além dos coeficientes de determinação $\left(R^{2}\right)$ estarem próximos à nulidade, assim, não podem ser usados para fins preditivos. Assim, os resultados indicam que nenhuma variável independente adotada (concentrações e tipo de antioxidantes) mostrou ter significância na diminuição de TBARS e no aumento da CR-F do ovo integral pasteurizado atomizado.

A equação de regressão mostra que os três antioxidantes adicionados ao ovo líquido integral pasteurizado influenciaram o aumento dos ácidos graxos livres, sendo que o $\mathrm{BHA}$, isoladamente reduz, e o alecrim e chá verde aumentam significativamente essa resposta. Além disso, a equação do modelo de regressão demonstra que somente o BHA e o alecrim afetaram as AS-233, reduzindo significativamente sua formação (Tabela 4).

Os resultados experimentais mostraram que a metodologia de superfície de resposta foi adequada para descrever a formação de AGL e AS-233 durante a atomização de ovo líquido integral pasteurizado, uma vez que o modelo matemático proposto foi significativo ( $p$ de regressão $<0,05$ ), com falta de ajuste não significativo $(p>0,05)$, e explicaram pelo modelo matemático $\left(R^{2}\right)$, respectivamente, 93,59 e $63,74 \%$ da variabilidade dos resultados, podendo então ser usados para fins preditivos (Tabela 4).

A RSM nem sempre é utilizada com sucesso para descrever resultados experimentais, ou seja, nem sempre se tem valores $R^{2}$ altos ou significância no modelo matemático (PAUCARMENACHO et al., 2008; OMWAMBA; HU, 2009; GRANATO et al., 2010; HOSSAIN et al., 2010; SAHA et. al, 2011). Isso é bastante comum, pois nem sempre é possível, por exemplo, colocar reações bioquímicas em uma equação matemática, já que muitos outros fatores, além dos testados, podem influenciar significativamente a resposta experimental.

$\mathrm{Na}$ Figura 1 encontram-se os resultados referentes à superfície de resposta da capacidade antioxidante total da fração lipídica (CATL) do ovo integral pasteurizado atomizado, em função das concentrações adicionadas de alecrim e chá verde. Observou-se que quanto menor a concentração dos antioxidantes, maior a CATL do ovo integral pasteurizado atomizado. Em maiores concentrações, o alecrim e o chá verde exerceram efeito pró-oxidante.

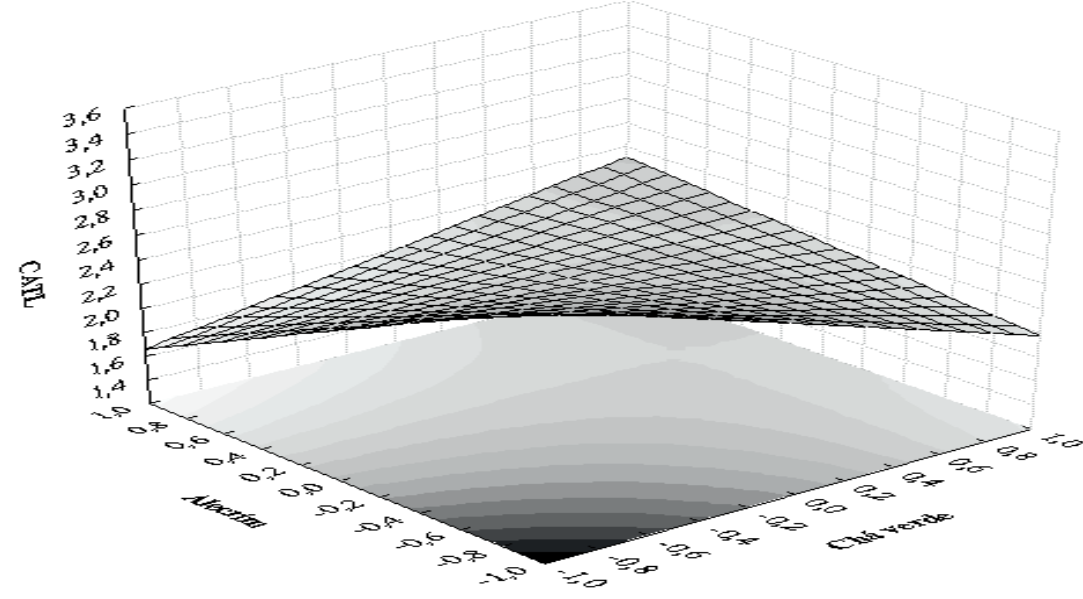


Na Figura 2 encontram-se os resultados referentes à superfície de resposta dos ácidos graxos livres ( $A G L$ ) do ovo integral pasteurizado atomizado, em função da concentração adicionada de chá verde e BHA. O chá verde e o BHA exerceram influência sobre a formação de AGL. Quanto maior foi à concentração dos antioxidantes, maior a formação dos ácidos graxos livres. Possivelmente, o chá verde e o BHA potencializaram a hidrólise de lipídios em ácidos graxos livres.

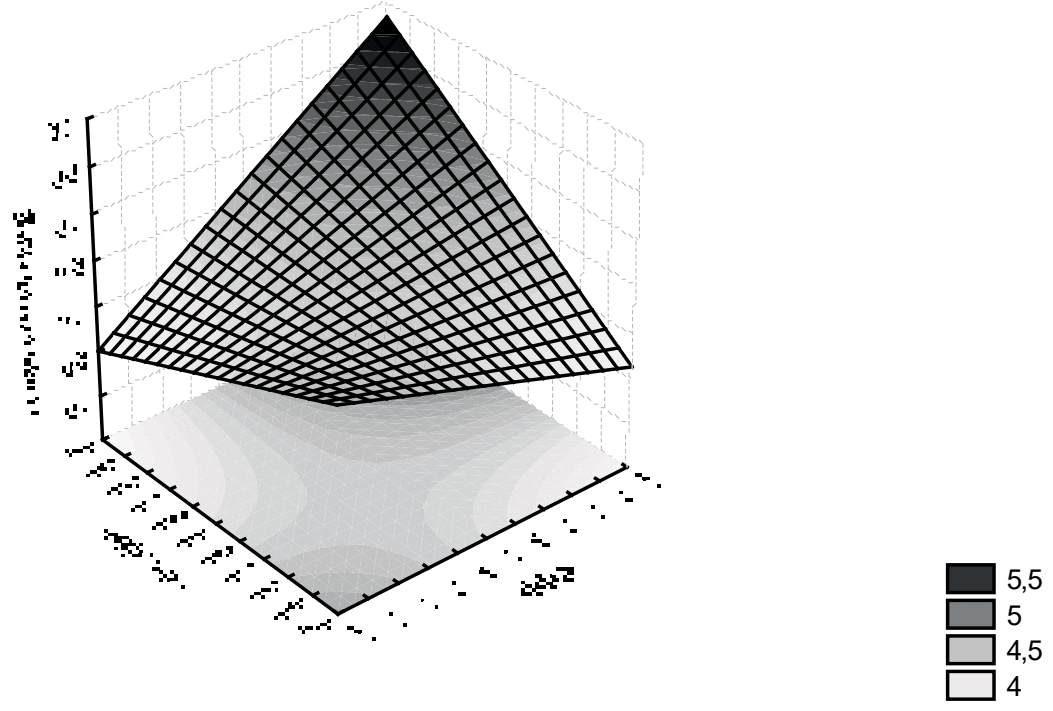

FIGURA 2. SUPERFÍCIE DE RESPOSTA DOS ÁCIDOS GRAXOS LIVRES (AGL: \% ÁCIDO OLÉICO/G LIPÍDIO) DO OVO INTEGRAL PASTEURIZADO ATOMIZADO, EM FUNÇÃO DA CONCENTRAÇÃO ADICIONADA DE CHÁ VERDE E BHA

$\mathrm{Na}$ Figura 3 encontram-se os resultados referentes à superfície de resposta dos ácidos graxos livres (AGL) do ovo integral pasteurizado atomizado, em função da concentração adicionada de alecrim e BHA. Quanto maior foi à concentração dos antioxidantes, maior a formação dos ácidos graxos livres. Assim como consta na Figura 2, possivelmente o alecrim e o BHA potencializaram a hidrólise de lipídios em ácidos graxos livres.

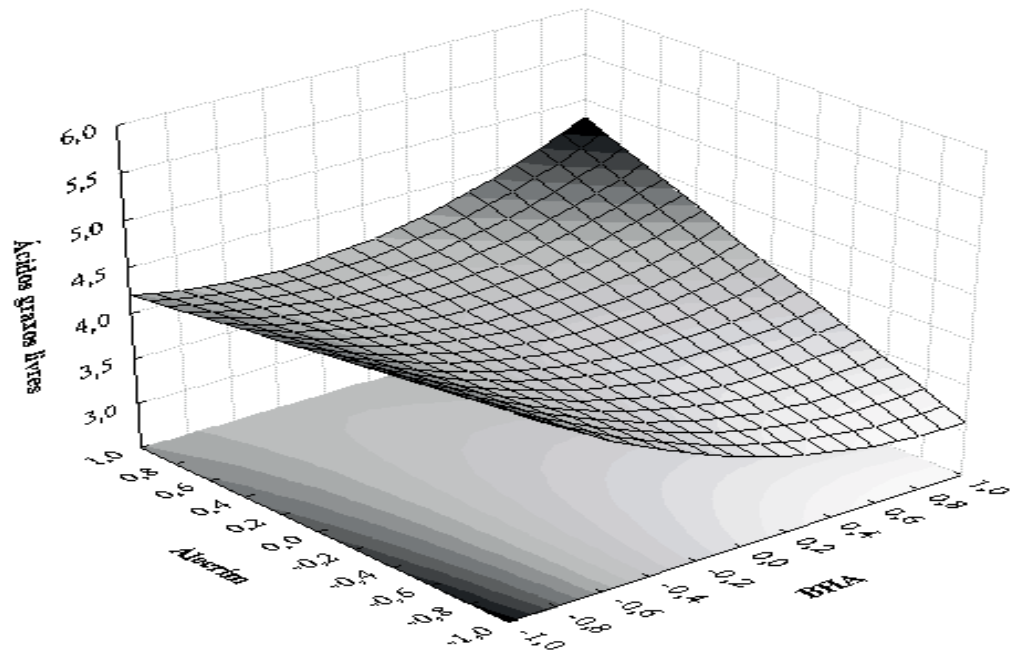

FIGURA 3. SUPERFÍCIE DE RESPOSTA DOS ÁCIDOS GRAXOS LIVRES (AGL: \% ÁCIDO OLÉICO/G LIPÍDIO) DO OVO INTEGRAL PASTEURIZADO ATOMIZADO, EM FUNÇÃO DA CONCENTRAÇÃO ADICIONADA DE ALECRIM E BHA 
Na Figura 4 encontra-se a superfície de resposta das substâncias absorvidas a 233nm (AS233) do ovo integral pasteurizado atomizado, em função da concentração adicionada de alecrim e BHA. O alecrim e o BHA exerceram influência sobre as AS-233. Quanto maior foi à concentração dos antioxidantes menor a formação das AS-233.
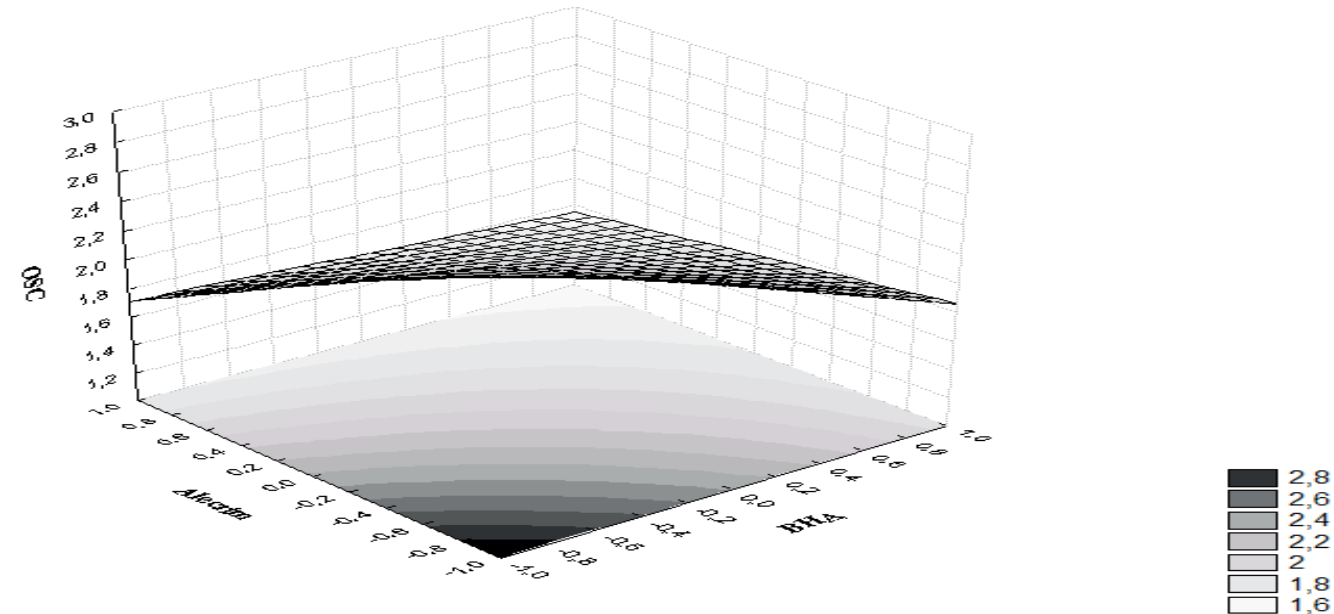

FIGURA 4. SUPERFÍCIE DE RESPOSTA DAS SUBSTÂNCIAS ABSORVIDAS A 233NM (AS-233: EQUIVALENTE EM MG 7-CETOCOLESTEROL/ MG LIPÍDIO) DO OVO INTEGRAL PASTEURIZADO ATOMIZADO, EM FUNÇÃO DA CONCENTRAÇÃO ADICIONADA DE ALECRIM E BHA

\subsection{OTIMIZAÇÃO DAS CONCENTRAÇÕES DE ANTIOXIDANTES}

Os resultados obtidos nas Tabelas 3 e 4 demonstraram que a metodologia de superfície de resposta (RSM) foi adequada apenas para descrever a formação de AGL e AS-233, podendo então ser usados para fins preditivos e, portanto otimizados. Embora o modelo matemático relativo aos ácidos graxos livres (AGL) pudesse ser usado para fins preditivos, à opção de não utilizá-lo na otimização, decorreu do fato de tais compostos não serem propriamente produtos gerados no processo oxidativo lipídico, ainda que sejam mais susceptíveis à oxidação na forma livre.

Realizou-se a otimização da concentração da mistura de antioxidantes visando à inibição e/ou diminuição na formação das substâncias absorvidas a 233nm (AS-233), com isso, a função de desejabilidade total foi de 0,59 e o sistema antioxidante proposto pela RSM de 150ppm de BHA, $600 \mathrm{ppm}$ de alecrim e 300ppm de chá verde, o que corresponde aos ensaios 9 e 10 do experimento realizado (Tabela 1).

Seria essa a composição ótima dos antioxidantes para diminuir a formação das AS-233 no ovo integral pasteurizado atomizado. O valor médio previsto pelo modelo matemático foi de 2,015 equivalente em $\mu \mathrm{g}$ 7-cetocolesterol/mg de lipídio. A partir disso, uma nova amostra (ovo líquido integral pasteurizado) contendo os valores mencionados de cada antioxidante foi formulada e submetida aos mesmos protocolos experimentais, para averiguar a adequabilidade dos modelos em seu poder preditivo.

O resultado médio experimental obtido foi 2,09 \pm 0,29 equivalente em $\mu \mathrm{g}$ 7-cetocolesterol/ $\mathrm{mg}$ de lipídio. Sendo assim, o erro absoluto residual entre os valores previstos e os experimentais foram calculados, resultando em $3,588 \%$, com valor-p $(0,7022)$ não significativo, aceitando, assim, a hipótese de nulidade, que evidencia a igualdade de médias entre o valor previsto pelo modelo matemático oriundo da RSM e a média de um experimento independente, usando as mesmas 
condições experimentais.

\section{CONCLUSÕES}

Nas condições adotadas no delineamento experimental utilizado para verificar a influência das variáveis independentes (extrato de alecrim, extrato de chá verde e BHA) sobre as propriedades físico-químicas do ovo integral pasteurizado atomizado, permitiu concluir que:

O efeito protetor da mistura dos antioxidantes, alecrim, chá verde e BHA, ao ovo integral pasteurizado atomizado exerceu influência positiva e/ou negativa sobre a estabilidade lipídica do ovo integral pasteurizado atomizado.

Foi encontrado um modelo matemático adequado e conveniente, por intermédio da metodologia de superfície de resposta (RSM), para descrever a formação das substâncias absorvidas a 233nm (As-233) durante a atomização do ovo líquido integral pasteurizado, podendo ser usado para fins preditivos.

A otimização visando à inibição e/ou diminuição na formação AS-233 durante a atomização do ovo líquido integral pasteurizado, mostrou um sistema antioxidante proposto pela RSM composto de 150ppm de BHA, 600ppm de alecrim e 300ppm de chá verde.

A RSM foi determinante para se constatar com precisão aceitável a influência das diferentes concentrações dos antioxidantes adicionados, com o objetivo de manter a estabilidade lipídica do ovo submetido à atomização.

\section{ABSTRAT \\ APPLICATION OF RESPONSE SURFACE METHODOLOGY IN OPTIMIZING THE BLEND OF ANTIOXIDANTS WITH EFFECT ON LIPID STABILITY OF ATOMIZED EGG ABSTRACT}

The egg to be a food rich in cholesterol and lipids becomes susceptible to oxidation, especially when subjected to processes involving high temperatures, such as atomisation. The prevention or reduction of lipid oxidation egg is possible by the use of antioxidants. The objectives of this work were: a) Verify through test concentration of antioxidants, lipid stability of pasteurized egg added atomized mixture of rosemary extract, green tea extract and BHA (AO) tea; b) Optimize the concentration of the mixture of $A O$ added the full pasteurized liquid egg, then atomized by the mathematical model proposed by Response Surface Methodology (RSM). Ten mixtures of antioxidants were tested in pasteurized egg atomized (OIPA) and reflected in its lipid oxidative stability, verified by the TBARS (thiobarbituric acid reactive substances), CATL (total antioxidant capacity of the lipid fraction), CT-F (reductive capacity by reactive Folin - Ciocalteau), FFA (free fatty acids) and AS-233 (substances absorbed to $233 \mathrm{~nm}$ ). The results showed that RSM was adequate to describe the formation of FFA and AS-233 in OIPA, the latter may be used for predictive purposes. The optimization of the mixture of antioxidants was based on the mathematical model obtained with the AS-233, which was proposed as an antioxidant BHA system 150ppm, 600ppm and 300ppm rosemary green tea. The RSM was crucial to be seen with acceptable accuracy the influence of different concentrations of antioxidants added to the egg to maintain its lipid stability.

KEYWORDS: EGG ATOMIZED, OXIDATION, ANTIOXIDANTS, RESPONSE SURFACE METHODOLO$G Y$

\section{REFERÊNCIAS}


AGÊNCIA NACIONAL DE VGILÂNCIA SANITÁRIA. Métodos Físico-Químicos para Análises de Alimentos. 4.ed. São Paulo: Instituto Adolfo Lutz; Brasília: Ministério da Saúde, 2005. 1020p. (Série A. Normas e manuais técnicos).

AFONSO, M. S.; SANTANA, L. S.; MANCINI-FILHO, J. Interação entre antioxidantes naturais e espécies reativas do oxigênio nas doenças cardiovasculares: perspectivas para a contribuição do alecrim (Rosmarinus offi cinalis L.). Nutrire: rev. Soc. Bras. Alim. Nutr. J. Brazilian Soc. Food Nutr., São Paulo, SP, v. 35, n. 1, p. 129-148, abr. 2010.

ANGELO, P.M.; JORGE, N. Compostos fenólicos em alimentos - Uma breve revisão. Rev. Inst. Adolfo Lutz, v. 66, n. 1, p.232-240, 2007

ANGELO, P. M.; JORGE, N. Avaliação do óleo de girassol adicionado de antioxidantes sob estocagem. Ciênc. Tecnol. Alimentos. v. 28, n. 2, Campinas, 2008.

BERTOLIN, T. E.; CENTENAR, A.; GIACOMELLI, B.; GIACOMELLI, F.; COLLA, L. M.; RODRIGUES, V. M. Antioxidantes naturais na prevenção da oxidação lipídica em charque de carne ovina. Braz. J. Food Technol., Campinas, v. 13, n. 2, p. 83-90, abr./jun. 2010.

BERTOLIN, T. E.; MARGARITES, A. C. F.; GIACOMELLI, B.; FRUETTI, A.; HORST, C.;TEIXEIRA, D. M. F. Ficocianina, tocoferol e ácido ascórbico na prevenção da oxidação lipídica em charque. Braz. J. Food Technol., Campinas, v. 14, n. 4, p. 301-307, out./dez. 2011.

BRASIL. Agência Nacional de Vigilância Sanitária (ANVISA). Resolução RDC n²3, de 15 de fevereiro de 2005 . Aprova "Regulamento Técnico que aprova o uso de aditivos alimentares, estabelecendo suas funções e seus limites máximos para a categoria de alimentos óleos e gorduras - subcategoria creme vegetal e margarinas". Diário Oficial da União, Brasília, 16 de fevereiro de 2005. Seção 1, p. 3.

CABONI, M.F.; BOSELLI, E.; MESSIA, M.C.; VELAZCO, V.; FRATIANNI, A.; PANFILI, G.; MARCONI, E. Effect of processing and storage on the chemical quality markers of spray-dried whole egg. J. Food Chem., Barking, v. 92, p. 293-303, 2005.

CAPITANI, C. D.; CARVALHO, A. C. L.; RIVELLI, D. P.; BARROS, S. B. M.; CASTRO, I. A. Evaluation of natural and synthetic compounds according to their antioxidant activity using a multivariate approach. European journal of lipid science and technology. v. 111, n. 11, p. 1090-1099, 2009.

CARVALHO, M. G. Influência do processamento, de antioxidantes e da estocagem sobre a estabilidade oxidativa lipídica do ovo. 2012. 155p. Tese (Doutorado) - Faculdade de Ciências Farmacêuticas-Universidade de São Paulo, São Paulo, 2012

CHEN, B. H.; CHEN, Y. C. Evaluation of the analysis of cholesterol oxides by liquid chromatography. J. chromatography A. Amsterdan, v. 661, p. $127-136,1994$.

CSALLANY, A.S.; KINDOM, S.E.; ADDIS, P.B.; LEE, J. HPLC method for quantitation of cholesterol and four of its major oxidation products in muscle and liver tissues. Lipids, Champaign, v. 24, n. 7, p. 645-651, 1989.

DANISCO. GUARDIAN® Natural Extracts. Disponível em: <http://www.danisco.com/products/product_range/antioxidants/ guardianr>Acesso em: 15/02/2011.

DEGÁSPARI, C. H.; WASZCZYNSKYJ J. N.; Propriedades antioxidantes de compostos fenólicos. Visão acadêmica. v. 5 , n.1, p.33-40, 2004

DERRINGER, G.; SUICH, R. Simultaneous optimisation of several response variables. Journal of Quality Technology, v. 12, p. 214-219, 1980.

ESCARABAJAL, C. Estabilidade oxidativa de colesterol em ovo líquido, ovo líquido pasteurizado e em ovo em pó atomizado, obtidos em laboratório. 2011. 106p. Tese (Doutorado) - Faculdade de Ciências Farmacêuticas Universidade de São Paulo, São Paulo, 2011.

FACCO, E. M. P.; LAGE, M. E.; GODOY, H. T. Influence of vitamin E supplemented diet on charque quality and lipid stabilization. Brazilian Archives of Biology and Technology. Curitiba, v. 52, n. 3, p. 729-736, 2009.

FELLOWS, P. J. Tecnologia do processamento de alimentos: princípios e prática, 2ed. Porto Alegre: Artmed, 2006. 602p.

FOLCH, J.; LEES, M.; STANLEY, G.H.S. A simple method for the isolation and purification of total lípides from animal tissues. J. Biol. Chem., Baltimore, v. 726, p. 497-509, 1957.

GALOBART, J.; BARROETA, A.C.; BAUCELLS,M.D.; GUARDIOLA, F. Lipid oxi dation in fresh and spray-dried eggs enriched with w3 and w6 polyunsaturated fatty acids during storage as affected by dietary vitamin $E$ and canthaxanthin supplementation. Poultry Science, v. 80, p. 327-337, 2001.

GRANATO, D.; CASTRO, I. A.; ELLENDERSEN, L. S. N.; MASSON, M. L. Physical Stability Assessment and Sensory Optimization of a Dairy-Free Emulsion Using Response Surface Methodology. Journal of food science. v. 75, n. 3, 2010.

GUARDIOLA, F.; CODONY, R.; RAFECAS, M.; GRAU, A.; JORDAN, A.; BOATELL, J. Oxysterol formation in spray-dried egg processed and stored under various conditions: prevention and relationship with other quality parameters. J. Agric. Food Chem., v. 25, p. 2229-2243, 1997. 
HOSSAIN, M. B.; BRUNTON, N. P.; MARTIN-DIANA, A. B.; BARRY-RYAN, C. Application of response surface methodology to optimize pressurized liquid extraction of antioxidant compounds from sage (Salvia officinalis L.), basil (Ocimum basilicum L.) and thyme (Thymus vulgaris L.). Food Function. v. 1, p. 269-277, 2010.

HUBBER, K.C.; PIKE, O.A.; HUBBER, C.S. Antioxidant inhibition of cholesterol oxidation in a spray-dried food system during accelerated storage. J. Food Sci. v. 60, n. 5, p. 909-916, 1995.

LAROSA, G. Desenvolvimento de produto cárneo de tilápia com antioxidantes naturais. 2011. 93p. Tese (Doutorado) - Faculdade de Ciências Farmacêuticas, Universidade Estadual Paulista. "Júlio de Mesquita Filho", Araraquara, 2011.

LAI, S. M.; GRAY, J. I.; BUCKLEY, D. J.; KELLY, P. M. Influence of Free Radicals and Other Factors on Formation of Cholesterol Oxidation Products in Spray-Dried Whole Egg. J. Agric. Food Chem., v. 43, n. 5, p. 1127-1131, 1995.

KITAHARA, S. E. Efeito do processamento e da estocagem sobre a formação de óxidos de colesterol em ovos. 2004. p.80. Tese (Doutorado) - Faculdade de Ciências Farmacêuticas-Universidade de São Paulo, São Paulo, 2004.

MAZALLI, M. R.; BRAGAGNOLO, N. Effect of Storage on Cholesterol Oxide Formation and Fatty Acid Alterations in Egg Powder. J. Agric. Food Chem. v. 55, p. 2743-2748, 2007.

MEDINA, M. K. J. Óxidos de colesterol em ovo em pó comercial: ocorrência e efeito do processamento e adição de tocoferóis no produto armazenado. 2009. 117p. Tese (Doutorado) - Faculdade de Ciências Farmacêuticas - Universidade de São Paulo, São Paulo, 2009.

MONTGOMERY, D. C. Design and analysis of experiments. New York: Wiley, 2000.

MOHAMED, R.; PINEDA, M.; AGUILAR, M. Antioxidant capacity of extracts from wild and crop plants of the mediterranean region. J. Food Science. v. 72, n.1, 2007.

MORAIS, S.M.; CAVALCANTI, E. S. B.; COSTA, S. M. O.; AGUIAR, L. A. Ação antioxidante de chás e condimentos de grande consumo no Brasil. Revista Brasileira de Farmacognosia, Brazilian Journal of Pharmacognosy. v. 19, n. 1, p. 315320, Jan./Mar. 2009.

MOURA, S. C. S. R.; GERMER, S. P. M.; ANJOS, V. D. A.; MORI, E. E. M.; MATTOSO, L. H.C.; FIRMINO, A.; NASCIMENTO, C. J. F. Influência dos parâmetros de torração nas características físicas, químicas e sensoriais do café arábica puro. Braz. J. Food Technol., Campinas, v. 10, n. 1, p. 17-25, jan./mar. 2007.

MORGAN, J.N.; ARMSTRONG, D.J. Formation of cholesterol-5,6-epoxides during spray-drying egg yolk. J. Food Sci. v. 52, p.1224-1227, 1987.

NAWAR, W. W. Lipids. p. 269 - 382. In: FENNEMA, O. R (Ed). Química de los alimentos, Zaragosa: Editoral Acribia S. A., $2000,1258 p$.

OHATA, S. M.; VIOTTO, L. A. Comportamento reológico de constituintes do ovo. Braz. J. Food Technol. Campinas, v. 14, n. 1, p. 10-18, jan./mar. 2011.

OMWAMBA, M.; HU, Q. Antioxidant capacity and antioxidative compounds in barley (Hordeum vulgare L.) grain optimized using response surface methodology in hot air roasting. Eur Food Res Technol. v. 229, p. 907-914, 2009.

PAUCAR-MENACHO, L. M.; SILVA, L. H.; BARRETTO, P. A. A.; MAZAL, G. FAKHOURI, F. M.; STEEL, C. J.; COLLARESQUEIROZ, F. P. Desenvolvimento de massa alimentícia fresca funcional com a adição de isolado protéico de soja e polidextrose utilizando páprica como corante. Ciênc. Tecnol. Alimentos. Campinas, v. 28, n. 4, p. 767-778, 2008.

PERUMALLA, A.V.S.; NAVAM. S. H. Green tea and grape seed extracts - Potential applications in food safety and quality. Food Research International. v. 44, p. 827-839, 2011.

PENAZZI, G.; CABONI, M. F.; ZUNIN, P.; EVANGELISTI, F.; TISCORNIA, E.; TOSCHI, T.G.; LERCKER, G. Routine highperformence liquid chromatographic determination of free 7-ketocholesterol in some foods by two different analytical methods, J. Am. Oil Chen. Soc, Champaign, v. 72, n. 2, p. 1523-1527, 1995.

PRIETO, P.; PINEDA, M.; AGUILAR, M. Spectrophotometric quantitation of antioxidant capacity through the formation of a phosphomolybdenum complex: specific application to the determination of vitamin E. Analytical Biochemistry. v. 269, n. 2, p. 337-341, 1999.

RAMALHO, V. C; JORGE, N. Antioxidantes utilizados em óleos, gorduras e alimentos gordurosos. Quim. Nov. v. 29, n. 4, p.755-760, 2006.

RIBEIRO, E. P.; SERAVALLI, E. A. G. Química de Alimentos. São Paulo: Edgard Blücher, 2004.

ROSA, E. D.; TSUKADA, M.; FREITAS, L. A. P. Secagem por atomização na indústria alimentícia: fundamentos e aplicações Disponível em: http://www.fcf.usp.br/ensino/graduacao/disciplinas/exclusivo/inserir/anexos/linkanexos/ secagem $\% 20$ de $\% 20$ materiais.pdf/ Acesso em: 07/06/2010.

SAS INSTITUTE. SAS software: application of statistical tests through release 6.12. Cary: Statistical Analysis System Institute, 
1997.

SAHA, J; DEBNATH, M.; SAHA, A.; GHOSH, T.; SARKAR, P. Response surface optimisation of extraction of antioxidants from strawberry fruit, and lipid peroxidation inhibitory potential of the fruit extract in cooked chicken patties. J Sci Food Agric. v. 91, p. $1759-1765,2011$.

TAI, C.Y.; CHEN, Y.C.; CHEN, B.H. Analysis, formation and inhibition of cholesterol oxidation products in foods: an overview (Part II). J. Food Drug Anal., Nankang, v.8, n.1, p.1-15, 2000.

TOMPKINS, C.; PERKINS, E. G. Frying performance of low-linolenic acid soybean oil. Journal American Oil Chemistry Society, Chicago, v. 77, n. 3, p. 223-229, 2000.

VYNCKE, B.W. Direct determination of the thiobarbituric acid value in trichloracetic acid extracts of fish as a measure of oxidative rancidity. Fette Seifen Anstrichmittbl, Leinfelden, v. 72, n.12, p.1084-1087, 1970.

ZHANG, Q.; ZHANG, J.; SHEN, J.; SILVA, A.; DENNIS, D. A.; BARROW, C. J. A simple 96-well microplate method for estimation of total polyphenolcontent in seaweeds. Journal of Applied Phycology. v. 18, p. 445-450, 2006.

ZHENG, W.; WANG, S. Y. Antioxidant activity and phenolic compounds in selected herbs. Journal of Agriculture and Food Chemistry, v. 49, n. 11, p. 5165-5170, 2001.

WU, Y.; LI, L.; DUAN, Z.; YANG, X.; SHANG, J.; CHEN, S. Application of Response Surface Methodology to Optimise Preparation High Antioxidant Activity Product from Pinctada Fucata Muscle. Advanced Materials Research, v. 396-398, p. 1341-1348, 2012.

\section{AGRADECIMENTOS}

A Universidade de São Paulo pelo apoio técnico e infraestrutura. A DANISCO pela concessão dos antioxidantes. Ao CNPq e FAPESP pelo apoio financeiro. 